\title{
Structure of a positive-parity band in ${ }^{130} \mathrm{Pr}$
}

\author{
K.Y. Ma ${ }^{1,2}$, J.B. Lu ${ }^{1, a}$, X. Xu ${ }^{1}$, Y.M. Liu ${ }^{1}$, Z. Zhang ${ }^{1}$, X.Y. Li ${ }^{1}$, D. Yang ${ }^{1}$, Y.Z. Liu ${ }^{1}$, X.G. Wu ${ }^{3}$, C.Y. He $^{3}$, \\ Y. Zheng ${ }^{3}$, and C.B. $\mathrm{Li}^{3}$ \\ 1 College of Physics, Jilin University, Changchun 130012, China \\ 2 College of Electronic Science \& Engineering, Jilin University, Changchun 130012, China \\ 3 China Institute of Atomic Energy, Beijing 102413, China
}

Received: 1 August 2016 / Revised: 3 January 2017

Published online: 23 January 2017

(C) The Author(s) 2017. This article is published with open access at Springerlink.com Communicated by A. Gade

\begin{abstract}
High-spin states of ${ }^{130} \mathrm{Pr}$ have been populated using the ${ }^{99} \mathrm{Ru}\left({ }^{35} \mathrm{Cl}, 2 \mathrm{n} 2 \mathrm{p}\right){ }^{130} \mathrm{Pr}$ reaction at a beam energy of $151 \mathrm{MeV}$. A new positive-parity side band has been identified and possible interpretations on the origin of the side band have been discussed.
\end{abstract}

\section{Introduction}

In the $A \sim 130$ mass region, the nearly degenerate $\Delta I=1$ doublet bands built on the $\pi h_{11 / 2} \otimes \nu h_{11 / 2}$ configuration have attracted significant attention and intensive discussion in odd-odd $N=77\left({ }^{132} \mathrm{Cs},{ }^{134} \mathrm{La}\right)[1,2]$, $N=75\left({ }^{130} \mathrm{Cs},{ }^{132} \mathrm{La},{ }^{134} \mathrm{Pr},{ }^{136} \mathrm{Pm},{ }^{138} \mathrm{Eu}\right)[3-6], N=73$ $\left({ }^{128} \mathrm{Cs},{ }^{130} \mathrm{La},{ }^{132} \mathrm{Pr}\right)[7,8], N=71\left({ }^{126} \mathrm{Cs},{ }^{128} \mathrm{La}\right)[9,10]$, $N=69\left({ }^{124} \mathrm{Cs},{ }^{126} \mathrm{La},{ }^{128} \mathrm{Pr}\right)[11-13]$ and $N=67\left({ }^{122} \mathrm{Cs}\right.$, $\left.{ }^{124} \mathrm{La}\right)[14,15]$ isotones. These $\Delta I=1$ doublet bands with the same positive parity are mostly interpreted as a manifestation of "chirality" in the sense of the angular momentum coupling [16].

Previously, we have reported the positive-parity doublet bands with the configuration of $\pi h_{11 / 2} \otimes \nu h_{11 / 2}$ in ${ }^{126} \mathrm{La} \mathrm{[12]} \mathrm{and}{ }^{128} \mathrm{La}$ [10] respectively. So far, the doublet bands have been systematically observed in odd-odd La isotopes from ${ }^{124} \mathrm{La}$ to ${ }^{134} \mathrm{La}$. However, for the Pr isotope chain, the doublet bands have been observed from ${ }^{128} \mathrm{Pr}$ to ${ }^{134} \mathrm{Pr}$ except for ${ }^{130} \mathrm{Pr}$. Meanwhile, the doublet bands have also been observed in the ${ }^{126} \mathrm{Cs}[9]$ and ${ }^{128} \mathrm{La} \mathrm{[10],}$ which are the isotones of ${ }^{130} \mathrm{Pr}$. Hence, we infer that similar bands may appear in ${ }^{130} \mathrm{Pr}$. The validity of theories is often tested by how well the theory reproduces the systematic behavior of isotopes or isotones, and thus it is important to establish the doublet bands that systematically appear in the Pr isotopic chain. Based on the above considerations, a new reaction different from the previous experiment of ${ }^{130} \operatorname{Pr}[17,18]$ is performed in the HI-13 tandem accelerator at CIAE in Beijing, and the main purpose of the experiment is to search for the positive-parity doublet bands.

\footnotetext{
a e-mail: ljb@jlu.edu.cn
}

\section{Experimental details}

High-spin states of ${ }^{130} \mathrm{Pr}$ were populated through the ${ }^{99} \mathrm{Ru}\left({ }^{35} \mathrm{Cl}, 2 \mathrm{n} 2 \mathrm{p}\right){ }^{130} \mathrm{Pr}$ reaction at a beam energy of 151 $\mathrm{MeV}$. The ${ }^{99} \mathrm{Ru}$ target, with an enrichment of $92.8 \%$ and a thickness of $2.24 \mathrm{mg} / \mathrm{cm}^{2}$, was rolled onto a $15 \mathrm{mg} / \mathrm{cm}^{2}$ lead backing. The beam was provided by the HI-13 tandem accelerator at CIAE in Beijing. The $\gamma-\gamma$ coincidence data were recorded by the use of the detecting system consisting of nine compton-suppressed HPGe detectors, two HPGe planar detectors and one clover-type detector. A total of $4.5 \times 10^{8} \gamma-\gamma$ coincidence events were recorded. The data were sorted into a symmetrized $\gamma-\gamma$ coincidence matrix and a DCO (directional correlation from oriented states) matrix. DCO ratios were obtained from spectra gated on quadrupole transitions. For our detector array, when gating on a quadrupole transition, the DCO ratio of the measured transition is around 1.0 for quadrupole transition and around 0.6 for dipole transition.

\section{Results and discussion}

The positive-parity yrast band 1 is the most intensely populated in the present experiment, and its configuration has been assigned to $\pi h_{11 / 2} \otimes \nu h_{11 / 2}$ in the previous work $[17,18]$. In the present work, two new 199.5 and $53.5 \mathrm{keV}$ transitions have been observed at the bottom of this band, and the spin of the lowest observed state is extended to $\left(6^{+}\right)$. Parities and spins of this band are assigned based on the proposed configuration and the systematics of Liu et al. [19].

Band 2 (side band) is reported for the first time, and several linking transitions between bands 1 and 2 are observed. Multipolarity analysis indicates that the 1080.5 
Table 1. Energies, intensities, and DCO ratio of $\gamma$-rays related to band 1 and band 2 and linking $\gamma$-rays between them in ${ }^{130} \mathrm{Pr}$. The internal conversion is not taken into account in the present work.

\begin{tabular}{|c|c|c|c|c|}
\hline$E_{\gamma}(\mathrm{keV})$ & $I_{\gamma}$ & $R_{D C O}^{(a)}$ & $I_{i}^{\pi} \rightarrow I_{f}^{\pi}$ & Multipolarity \\
\hline \multicolumn{5}{|l|}{ Band 1} \\
\hline 53.5 & & & $7^{+} \rightarrow\left(6^{+}\right)$ & $(M 1 / E 2)$ \\
\hline 89.5 & $76.6(77)$ & $0.51(13)$ & $8^{+} \rightarrow 7^{+}$ & $M 1 / E 2$ \\
\hline 110.0 & $98.1(49)$ & $0.56(8)$ & $9^{+} \rightarrow 8^{+}$ & $M 1 / E 2$ \\
\hline $131.5^{(b)}$ & $100.0(25)$ & $0.58(7)$ & $10^{+} \rightarrow 9^{+}$ & $M 1 / E 2$ \\
\hline 143.5 & $12.5(38)$ & & $8^{+} \rightarrow\left(6^{+}\right)$ & $(E 2)$ \\
\hline 199.5 & $11.1(3.2)$ & $0.91(34)$ & $9^{+} \rightarrow 7^{+}$ & $E 2$ \\
\hline 213.7 & $61.5(61)$ & $0.62(12)$ & $12^{+} \rightarrow 11^{+}$ & $M 1 / E 2$ \\
\hline 226.5 & $82.2(51)$ & $0.57(11)$ & $11^{+} \rightarrow 10^{+}$ & $M 1 / E 2$ \\
\hline 242.0 & $13.1(39)$ & $0.92(36)$ & $10^{+} \rightarrow 8^{+}$ & $E 2$ \\
\hline 290.6 & $23.2(69)$ & $0.55(17)$ & $14^{+} \rightarrow 13^{+}$ & $M 1 / E 2$ \\
\hline 321.8 & $41.2(61)$ & $0.59(16)$ & $13^{+} \rightarrow 12^{+}$ & $M 1 / E 2$ \\
\hline 358.0 & $20.2(60)$ & $1.12(34)$ & $11^{+} \rightarrow 9^{+}$ & $E 2$ \\
\hline 362.0 & $13.8(39)$ & $0.51(19)$ & $16^{+} \rightarrow 15^{+}$ & $M 1 / E 2$ \\
\hline 386.6 & $23.0(69)$ & $0.54(17)$ & $15^{+} \rightarrow 14^{+}$ & $M 1 / E 2$ \\
\hline 419.7 & $7.4(21)$ & $0.68(26)$ & $18^{+} \rightarrow 17^{+}$ & $M 1 / E 2$ \\
\hline 422.1 & $12.5(36)$ & $0.65(23)$ & $17^{+} \rightarrow 16^{+}$ & $M 1 / E 2$ \\
\hline 428.1 & $6.1(18)$ & $0.52(22)$ & $19^{+} \rightarrow 18^{+}$ & $M 1 / E 2$ \\
\hline 440.4 & $40.7(61)$ & $1.05(25)$ & $12^{+} \rightarrow 10^{+}$ & $E 2$ \\
\hline 535.5 & $23.1(69)$ & $1.08(33)$ & $13^{+} \rightarrow 11^{+}$ & $E 2$ \\
\hline 612.2 & $48.0(72)$ & $0.98(24)$ & $14^{+} \rightarrow 12^{+}$ & $E 2$ \\
\hline 677.0 & $25.3(75)$ & $0.95(30)$ & $15^{+} \rightarrow 13^{+}$ & $E 2$ \\
\hline 748.5 & $35.1(70)$ & $1.12(34)$ & $16^{+} \rightarrow 14^{+}$ & $E 2$ \\
\hline 784.0 & $24.2(71)$ & $1.07(35)$ & $17^{+} \rightarrow 15^{+}$ & $E 2$ \\
\hline 842.0 & $26.0(76)$ & $0.92(31)$ & $18^{+} \rightarrow 16^{+}$ & $E 2$ \\
\hline 847.6 & $18.1(54)$ & & $19^{+} \rightarrow 17^{+}$ & $(E 2)$ \\
\hline 890.5 & $13.0(39)$ & $0.89(32)$ & $20^{+} \rightarrow 18^{+}$ & $E 2$ \\
\hline 891.5 & $14.0(42)$ & $0.91(30)$ & $21^{+} \rightarrow 19^{+}$ & $E 2$ \\
\hline \multicolumn{5}{|l|}{ Band 2} \\
\hline 241.5 & & & $12^{+} \rightarrow 11^{+}$ & $(M 1 / E 2)$ \\
\hline 264.1 & $2.5(11)$ & $0.65(28)$ & $13^{+} \rightarrow 12^{+}$ & $M 1 / E 2$ \\
\hline 272.5 & $4.0(13)$ & $0.54(24)$ & $14^{+} \rightarrow 13^{+}$ & $M 1 / E 2$ \\
\hline 321.5 & $1.3(8)$ & & $16^{+} \rightarrow 15^{+}$ & $(M 1 / E 2)$ \\
\hline 332.0 & $6.8(13)$ & $0.65(28)$ & $15^{+} \rightarrow 14^{+}$ & $M 1 / E 2$ \\
\hline 505.5 & $3.1(11)$ & $0.85(36)$ & $13^{+} \rightarrow 11^{+}$ & $E 2$ \\
\hline 536.6 & $4.4(14)$ & $0.87(37)$ & $14^{+} \rightarrow 12^{+}$ & $E 2$ \\
\hline 604.6 & $7.1(25)$ & $1.09(33)$ & $15^{+} \rightarrow 13^{+}$ & $E 2$ \\
\hline 653.5 & $3.4(13)$ & $1.15(42)$ & $16^{+} \rightarrow 14^{+}$ & $E 2$ \\
\hline 708.5 & & & $17^{+} \rightarrow 15^{+}$ & $(E 2)$ \\
\hline 768.0 & & & $18^{+} \rightarrow 16^{+}$ & $(E 2)$ \\
\hline
\end{tabular}

Linking

transitions

\begin{tabular}{lcclc}
\hline 1015.5 & $3.5(12)$ & $0.67(27)$ & $11^{+} \rightarrow 10^{+}$ & $M 1 / E 2$ \\
1030.0 & $2.5(12)$ & $0.53(25)$ & $12^{+} \rightarrow 11^{+}$ & $M 1 / E 2$ \\
1080.5 & $5.8(11)$ & $0.57(24)$ & $13^{+} \rightarrow 12^{+}$ & $M 1 / E 2$ \\
1294.0 & $2.3(7)$ & $1.09(45)$ & $13^{+} \rightarrow 11^{+}$ & $E 2$ \\
\hline
\end{tabular}

(a) DCO ratios listed here are obtained by setting the gate on quadrupole transitions.

(b) $I_{\gamma}$ are normalized to the $131.5 \mathrm{keV} \gamma$-ray in band 1 as 100

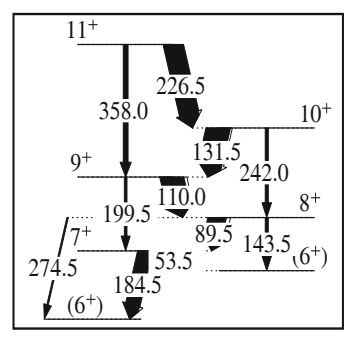

Band 1

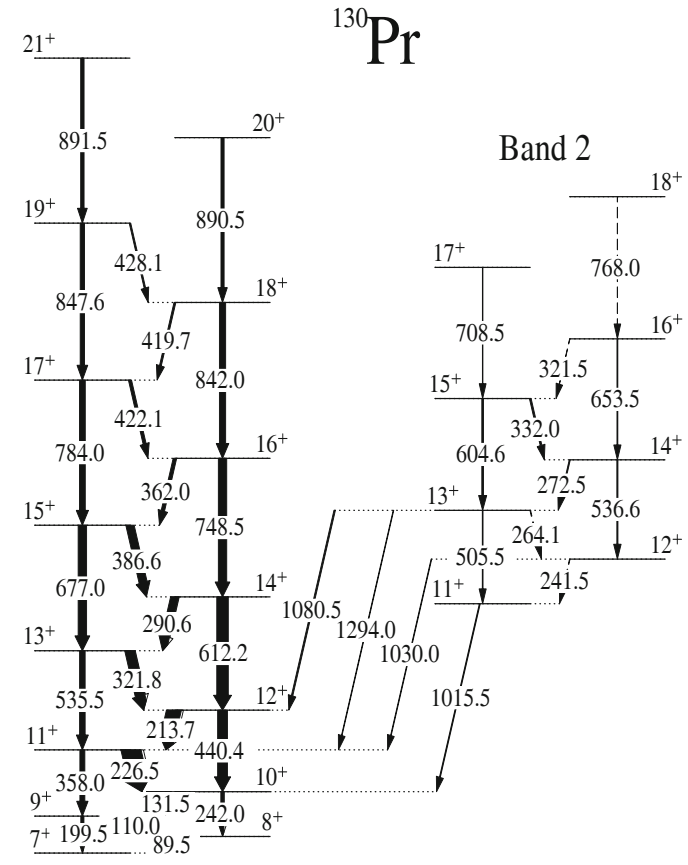

Fig. 1. Partial level scheme of ${ }^{130} \mathrm{Pr}$. The insertion shows the bottom portion of band 1 .

and $1030.0 \mathrm{keV}$ linking transitions are of $\Delta I=1$ character with DCO ratios of 0.57 and 0.53 respectively, and the $1294.0 \mathrm{keV}$ linking transition is of $\Delta I=2$ character with DCO ratio of 1.09 (DCO ratios from gating on the quadrupole transition). The observation of both $\Delta I=1$ and $\Delta I=2$ linking transitions between bands 1 and 2 implies that band 2 has positive parity as that of band 1. Meanwhile, the energies and spins of band 2 are fixed relative to the levels of band 1 as shown in fig. 1 . Properties and placements of related $\gamma$-rays are listed in table 1 . A sample $\gamma-\gamma$ coincidence spectrum supporting the level scheme of fig. 1 is shown in fig. 2 .

As mentioned above, the configuration of the yrast band (band 1) had previously been assigned as $\pi h_{11 / 2} \otimes$ $\nu h_{11 / 2}[17,18]$. In order to discuss the configuration assignment of the side band (band 2), cranked shell model (CSM) calculations [20,21] have been performed as shown in fig. 3, where the CSM calculations predict that proton $\omega_{E F}$ alignment occurs at $\sim 0.34 \mathrm{MeV}$. However, no crossing is found below $0.4 \mathrm{MeV}$ for bands 1 and 2 in the 


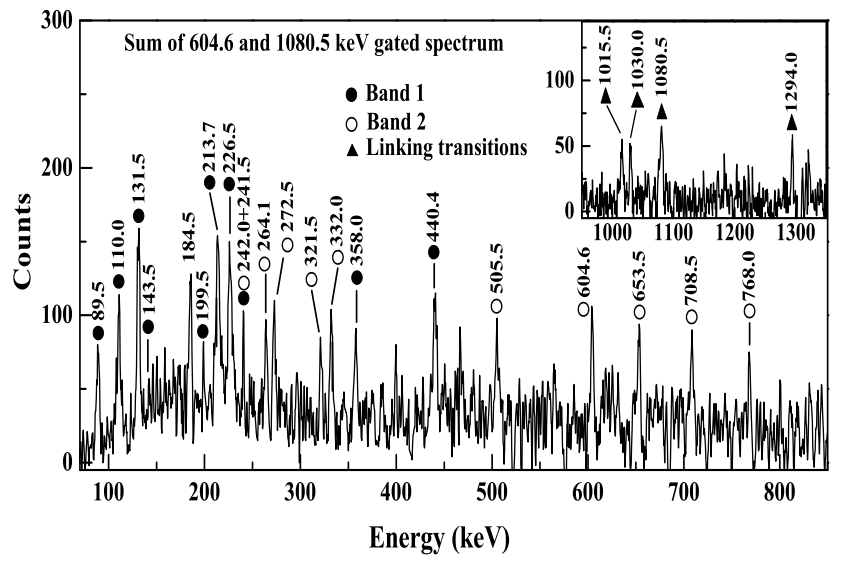

Fig. 2. Sample $\gamma-\gamma$ coincidence spectrum supporting the partial level scheme of ${ }^{130} \mathrm{Pr}$ as shown in fig. 1.

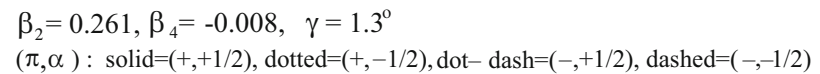

$(\pi, \alpha)$ : solid $=(+,+1 / 2)$, dotted $=(+,-1 / 2)$, dot - dash $=(-,+1 / 2)$, dashed $=(-,-1 / 2)$

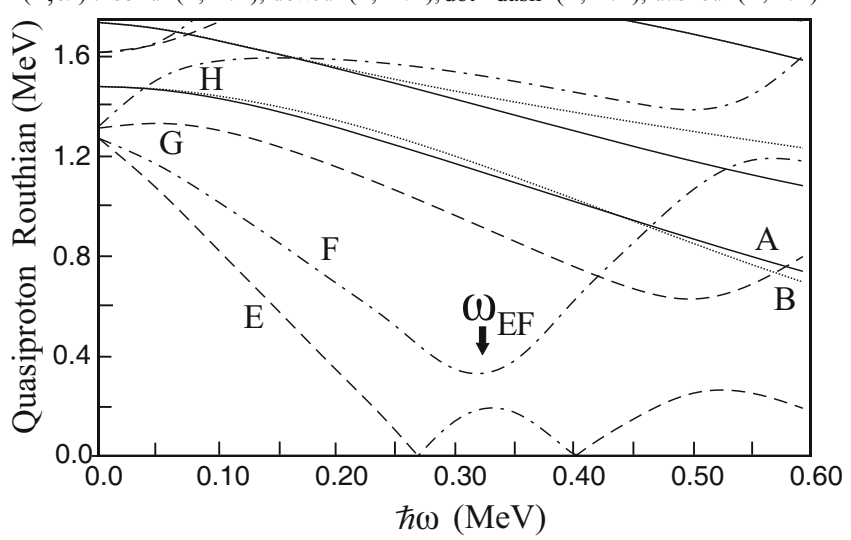

Fig. 3. Cranked shell model calculations for quasiproton Routhian. The deformation parameters shown at the top of the figure are determined by TRS calculations [22-24]. Interpretation of the lines is displayed at the top of the figure.

alignment plot (fig. 4(a)), indicating that the $h_{11 / 2}$ proton should be involved in the configurations of bands 1 and 2 to block the proton EF crossing. Moreover, the large initial alignments $(\sim 7 \hbar)$ for both bands strongly suggest that the $h_{11 / 2}$ neutron is involved in the configurations of bands 1 and 2. In addition, the experimental $B(M 1) / B(E 2)$ ratios for bands 1 and 2 are shown in fig. 4(b), along with the theoretical estimates of the geometrical model for the $\pi h_{11 / 2} \otimes \nu h_{11 / 2}$ configuration for comparison [25]. An agreement between the experiment and the theory is achieved for bands 1 and 2, also supporting the $\pi h_{11 / 2} \otimes \nu h_{11 / 2}$ configuration assignments for the two bands. However, it is difficult to assign a configuration for band 2 with the present data. The merits of several possible configurations for this sequence are considered below.

A second $\pi h_{11 / 2} \otimes \nu h_{11 / 2}$ band (side band) has been observed in many odd-odd nuclei in the $A \sim 130$ mass region, while the yrast band of these odd-odd nuclei is also assigned to the $\pi h_{11 / 2} \otimes \nu h_{11 / 2}$ configuration.

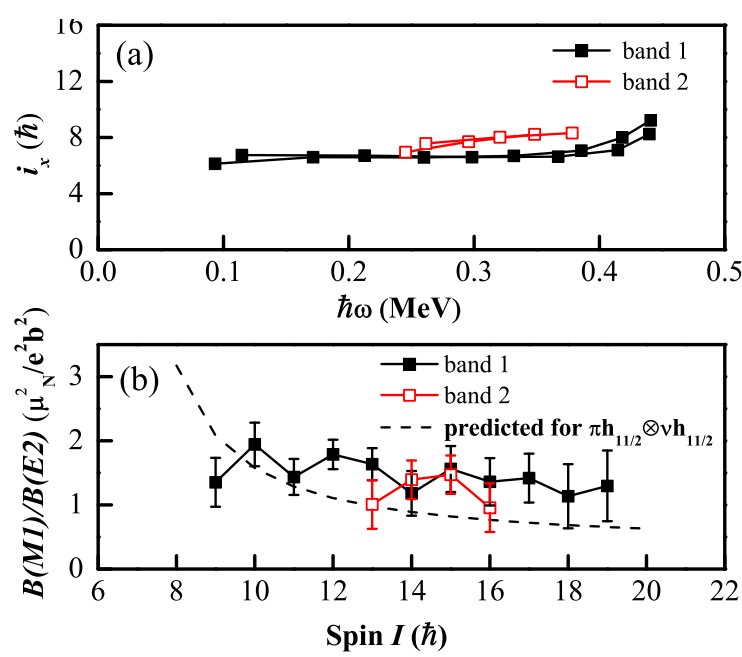

Fig. 4. (Color online) (a) Experimental alignment plots for bands 1 and 2 in ${ }^{130} \mathrm{Pr}$. The Harris parameters are $J_{0}=$ $19.4 \mathrm{MeV}^{-1} \hbar^{2}, J_{1}=29.3 \mathrm{MeV}^{-3} \hbar^{4}$ [17]. (b) Comparison of experimental and predicted $B(M 1) / B(E 2)$ values for bands 1 and 2 .

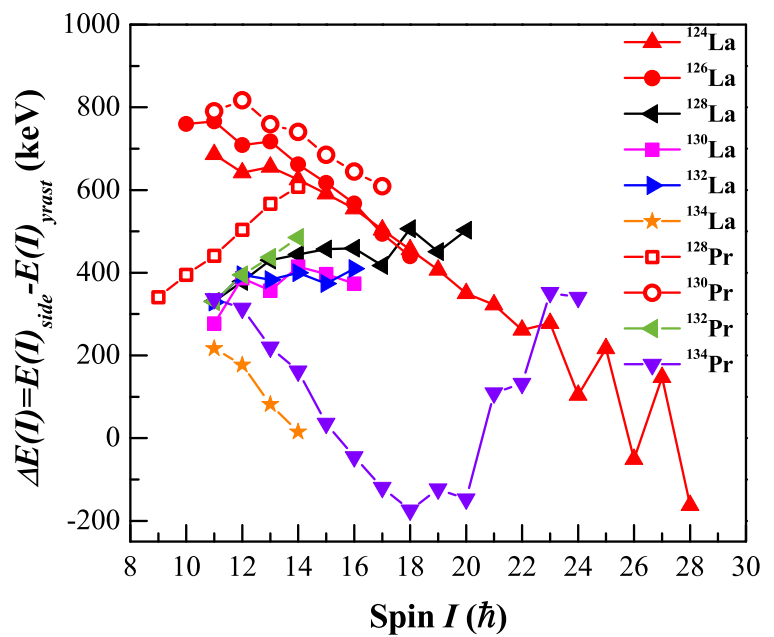

Fig. 5. (Color online) $\Delta E(I)=E(I)_{\text {side }}-E(I)_{\text {yrast }}$ of the two bands in ${ }^{130} \mathrm{Pr}$ compared with those in ${ }^{124} \mathrm{La}[15],{ }^{126} \mathrm{La}[12]$ ${ }^{128} \mathrm{La}[10],{ }^{130} \mathrm{La}[8],{ }^{132} \mathrm{La}[3],{ }^{134} \mathrm{La}[2],{ }^{128} \operatorname{Pr}[13],{ }^{132} \operatorname{Pr}[8]$ and ${ }^{134} \operatorname{Pr}[4]$.

The separation energies between the side band and the yrast band at the same spin, $\Delta E(I)=E(I)_{\text {side }}-$ $E(I)_{\text {yrast }}$, of ${ }^{130} \mathrm{Pr}$ together with those of some other La and $\mathrm{Pr}$ isotopes are presented in fig. 5. $\Delta E(I)$ of these oddodd nuclei fall into two groups. The two bands of odd-odd nuclei belonging to the lower group have been interpreted as nearly degenerate chiral doublet bands resulting chiral symmetry breaking. As shown in fig. 5 , the $\Delta E(I)$ of ${ }^{124} \mathrm{La} \mathrm{[15],}{ }^{126} \mathrm{La} \mathrm{[12]} \mathrm{and}{ }^{130} \mathrm{Pr}$ of the present work is too large for interpreting the two bands in ${ }^{124} \mathrm{La},{ }^{126} \mathrm{La}$ and ${ }^{130} \mathrm{Pr}$ as nearly degenerate chiral doublet band.

The magnitude of $\Delta E(I)$ in ${ }^{124} \mathrm{La}[15],{ }^{126} \mathrm{La} \mathrm{[12]} \mathrm{and}$ ${ }^{130} \mathrm{Pr}$ are similar and they decrease with increasing spin. The alignments of the side band and yrast band in ${ }^{124} \mathrm{La}$ and ${ }^{126} \mathrm{La}$ are also very similar to those of ${ }^{130} \mathrm{Pr}$. The 
alignments of the side band are $\sim 1.5 \hbar$ greater than that of the yrast band below $0.4 \mathrm{MeV}(\hbar \omega)$ for ${ }^{124} \mathrm{La},{ }^{126} \mathrm{La}$ and ${ }^{130} \mathrm{Pr}$ (see fig. 11(b) for ${ }^{124} \mathrm{La} \mathrm{[15]}$, and fig. 4(a) for ${ }^{126} \mathrm{La} \mathrm{[12])}$. In the cases of ${ }^{124} \mathrm{La}[15]$ and ${ }^{126} \mathrm{La}[12]$, the side band has been interpreted as resulting from the coupling between the unfavored signature of the $h_{11 / 2}$ proton and the two signatures of the $h_{11 / 2}$ neutron, while the yrast $\pi h_{11 / 2} \otimes \nu h_{11 / 2}$ band is interpreted as resulting from the coupling between the favored signature of the $h_{11 / 2}$ proton and the two signatures of the $h_{11 / 2}$ neutron. The alignment is the negative slope of the Routhian. From fig. 3 one sees that the slope of the F trajectory (unfavored signature of the $h_{11 / 2}$ proton) is smaller than that of the E trajectory (favored signature of the $h_{11 / 2}$ proton) leading to smaller alignments of the band 2 than those of the yrast band. Instead, the alignment of band 2 (see fig. $4(\mathrm{a}))$ is higher than that of the yrast structure. Additionally, fig. 3 indicates that the energy difference between the $\mathrm{F}$ trajectory and the E trajectory increases with $\hbar \omega$. In contrast, the $\Delta E(I)$ of ${ }^{124} \mathrm{La}[15],{ }^{126} \mathrm{La}[12]$ and ${ }^{130} \mathrm{Pr}$ decreases with increasing spin as shown in fig. 5 . Therefore, interpreting the side band in ${ }^{124} \mathrm{La} \mathrm{[15]} \mathrm{and}{ }^{126} \mathrm{La} \mathrm{[12}$ as resulting from the coupling between the unfavored signature of the $h_{11 / 2}$ proton and the two signatures of the $h_{11 / 2}$ neutron has to be reconsidered, and such a interpretation is not applicable to the case of ${ }^{130} \mathrm{Pr}$ either. Indeed, the $\pi h_{11 / 2} \otimes \nu h_{11 / 2}$ side band observed in ${ }^{128} \operatorname{Pr}$ (band 3 of fig. 1 in [13]) has been interpreted as resulting from the coupling between the unfavored signature of the $h_{11 / 2}$ proton and the two signatures of the $h_{11 / 2}$ neutron, and as expected the side band does have smaller alignments than those of the yrast band as shown in fig. 8(a) of [13], and the $\Delta E(I)=E(I)_{\text {side }}-E(I)_{\text {yrast }}$ does increase with spin as shown in fig. 5 .

$\gamma$-vibrational bands have been observed in even-even and odd- $A$ nuclei in various mass regions. In the nearby even-even nucleus, ${ }^{128} \mathrm{Ce}[26]$, the $\gamma$-vibrational band is observed approximately $700 \mathrm{keV}$ above the ground-state band, which is similar to the excitation of band 2 relative to the yrast band in ${ }^{130} \mathrm{Pr}$ as shown in fig. 1 . In addition, the $\gamma$-vibrational band in ${ }^{128} \mathrm{Ce}$ has slightly more alignment than the ground-state band at lower frequencies as shown in fig. 5(a) of ref. [26], which is consistent with that between band 2 and the yrast band in ${ }^{130} \mathrm{Pr}$ of the present study. Both of these features suggest that it is possible that band 2 is the $\gamma$-vibrational band resulted from the coupling between the yrast $\pi h_{11 / 2} \otimes \nu h_{11 / 2}$ band and a phonon. However, because the band-heads of the yrast band and the $\gamma$-vibrational band (band 2) are not observed, we are not able to provide further supporting evidences in terms of the discussions on the decay pattern of the band-head of the $\gamma$-vibrational band and energy difference between the band-heads of the two bands.

The fact that the band 2 in ${ }^{130} \mathrm{Pr}$ is not observed until high excitation energy $(>1347 \mathrm{keV})$, suggests that it is possible that band 2 is a four quasiparticles band. The alignment of the band 2 is $\sim 1.5 \hbar$ greater than that of the yrast band at lower frequencies, which suggests that except the $h_{11 / 2}$ proton and $h_{11 / 2}$ neutron, the other two nucleons should have very small alignment associated with them. However, due to the fact that only eight levels are observed in the side band, we are not able to make a reliable judgement on which two quasiparticles are coupled to the $h_{11 / 2}$ proton and $h_{11 / 2}$ neutron to form the side band.

To distinguish the above two possible interpretations of the band 2 in ${ }^{130} \mathrm{Pr}$, further experimental and theoretical studies are desirable.

\section{Summary}

High-spin states of ${ }^{130} \mathrm{Pr}$ have been populated using the ${ }^{99} \mathrm{Ru}\left({ }^{35} \mathrm{Cl}, 2 \mathrm{n} 2 \mathrm{p}\right){ }^{130} \mathrm{Pr}$ reaction at a beam energy of $151 \mathrm{MeV}$. A new positive-parity side band feeding into the yrast band has been observed and possible interpretations on the origin of the side band have been discussed. In the course of the study on the positive-parity side band in ${ }^{130} \mathrm{Pr}$, we noted that the $\pi h_{11 / 2} \otimes \nu h_{11 / 2}$ side band, previously reported in ${ }^{124} \mathrm{La}$ and ${ }^{126} \mathrm{La}$, had been interpreted as resulting from the coupling between the unfavored signature of the $h_{11 / 2}$ proton and the two signatures of the $h_{11 / 2}$ neutron, while the $\pi h_{11 / 2} \otimes \nu h_{11 / 2}$ yrast band was resulting from the coupling between the favored signature of the $h_{11 / 2}$ proton and the two signatures of the $h_{11 / 2}$ neutron. Such interpretation is not supported by the arguments presented in the present study.

This work is supported by the National Natural Science Foundation of China under Grant Nos. 11405072, 11075064, and 11205069, China Postdoctoral Science Foundation Nos. 2015M571354 and 2013M541285, the National Basic Research Programme of China under Grant Nos. 2007CB815005, and the Specialized Research Fund for the Doctoral Programme of Higher Education of China under Grant No. 20050183008.

Open Access This is an open access article distributed under the terms of the Creative Commons Attribution License (http://creativecommons.org/licenses/by/4.0), which permits unrestricted use, distribution, and reproduction in any medium, provided the original work is properly cited.

\section{References}

1. G. Rainovski et al., Phys. Rev. C 68, 024318 (2003).

2. R.A. Bark, A.M. Baxter, A.P. Byrne, G.D. Dracoulis, T. Kibédi, T.R. McGoram, S.M. Mullins, Nucl. Phys. A 691, 577 (2001).

3. K. Starosta et al., Phys. Rev. Lett. 86, 971 (2001).

4. C.M. Petrache et al., Nucl. Phys. A 597, 106 (1996).

5. J. Timar et al., Phys. Rev. C 84, 044302 (2011).

6. A.A. Hecht et al., Phys. Rev. C 63, 051302 (2001).

7. T. Koike, K. Starosta, C.J. Chiara, D.B. Fossan, D.R. LaFosse, Phys. Rev. C 67, 044319 (2003).

8. T. Koike, K. Starosta, C.J. Chiara, D.B. Fossan, D.R. LaFosse, Phys. Rev. C 63, 061304 (2001).

9. S.Y. Wang, Y.Z. Liu, T. Komatsubara, Y.J. Ma, Y.H. Zhang, Phys. Rev. C 74, 017302 (2006).

10. K.Y. Ma, J.B. Lu, D. Yang, H.D. Wang, Y.Z. Liu, X.G. Wu, Y. Zheng, C.Y. He, Phys. Rev. C 85, 037301 (2012). 
11. A. Gizon et al., Nucl. Phys. A 694, 63 (2001).

12. K.Y. Ma, J.B. Lu, S.P. Ruan, D. Yang, J. Li, Y.Z. Liu, Y.J. Ma, X.G. Wu, Y. Zheng, C.Y. He, Phys. Rev. C 88, 057302 (2013).

13. D.J. Hartley et al., Phys. Rev. C 65, 044329 (2002).

14. U. Yong-Nam et al., J. Phys. G 31, B1 (2005).

15. H.J. Chantler et al., Phys. Rev. C 66, 014311 (2002).

16. S. Frauendorf, J. Meng, Nucl. Phys. A 617, 131 (1997).

17. R. Ma et al., Phys. Rev. C 37, 1926 (1988).

18. C.M. Petrache et al., Nucl. Phys. A 635, 361 (1998).

19. Y.Z. Liu, J.B. Lu, Y.J. Ma, S.G. Zhou, H. Zheng, Phys. Rev. C 54, 719 (1996).
20. W. Nazarewicz, J. Dudek, R. Bengtsson, I. Ragnarsson, Nucl. Phys. A 435, 397 (1985).

21. S. Cwiok, J. Dudek, W. Nazarewicz, W. Skalski, T. Werner, Comput. Phys. Commun. 46, 379 (1987).

22. R. Wyss, J. Nyberg, A. Johnson, R. Bengtsson, W. Nazarewicz, Phys. Lett. B 215, 211 (1988).

23. W. Nazarewicz, G.A. Leander, J. Dudek, Nucl. Phys. A 467, 437 (1987).

24. W. Nazarewicz, R. Wyss, A. Johnson, Nucl. Phys. A 503, 285 (1989).

25. F. Dönau, Nucl. Phys. A 471, 469 (1987).

26. E.S. Paul et al., Nucl. Phys. A 676, 32 (2000). 